\title{
Synthesis of crosslinked poly(styrene-co-divinylbenzene-co-sulfopropyl methacrylate) nanoparticles by emulsion polymerization: Tuning the particle size and surface charge density
}

\author{
DHAMODARAN ARUNBABU, MOUSUMI HAZARIKA, SOMSANKAR NAIK and \\ TUSHAR JANA* \\ School of Chemistry, University of Hyderabad, Hyderabad 500 046, India
}

MS received 21 August 2008; revised 5 November 2009

\begin{abstract}
We have synthesized highly charged, crosslinked poly (styrene-co-divinylbenzene-co-sulfopropyl methacrylate) copolymer colloidal particles using emulsion polymerization. The effects of concentration of the emulsifier and the initiator on the particle size and the charge density of the colloidal particles are studied. Colloidal particle size is highly dependent upon the concentration of the emulsifier and the initiator. The colloidal particle diameter decreases with increasing concentration of the emulsifier and increases with increasing concentration of the initiator in the polymerization mixture. Number of particles, surface charge density and charges per particle are also functions of both the emulsifier and the initiator concentration. The surface charge density and the number of charges per sphere increase with increasing particle diameter. These copolymer colloid particles self assemble readily and diffract visible light. Polymer hydrogel imbibed with these colloids shows the light diffraction.
\end{abstract}

Keywords. Colloidal particle; emulsion polymerization; polystyrene; critical micelle concentration (CMC); surface charge density; charges per particle.

\section{Introduction}

Monodisperse polymer colloidal particles with narrow molecular weight distribution have unique properties which make them interesting and find applications in numerous fields (Fitch 1997; Xia et al 2000). Generally, emulsion polymerization technique is employed to prepare the monodisperse polymer particles with narrow molecular weight distribution (Odian 2004; Reese et al 2000; Reese and Asher 2002). Functionalization of polymer particles with the desired functional groups is obtained by employing appropriate ionic or nonionic monomers as a comonomer in the polymerization recipe. Functional polymer particles are useful in various fields such as catalysis, photonics (Luck et al 1963; Joannopoulos et al 1995; Soukoulis 1996, 1999), paint technology, medicine, biotechnology etc. Colloidal particles which are functionalized by ionic monomers can readily self assemble into non-close pack structures. These particles diffract light according to Bragg's law in the near-IR, ultra-violet and visible regions depending upon the particle size and volume fraction of the particles in the medium. This class of colloidal material is known as the crystalline colloidal

*Author for correspondence

(tjsc@uohyd.ernet.in; tjscuoh@gmail.com) arrays (CCA) (Krieger and O'Neil 1968; Hiltner and Krieger 1969; Hiltner et al 1971; Carlson and Asher 1984; Aastuen et al 1986; Rundquist et al 1989, 1991; Asher et al 1994). Asher et al (1994) has pioneered a new class of materials in which CCA are embedded inside polymer hydrogel matrix. This CCA polymerized polymer hydrogel is known as polymerized crystalline colloidal array (PCCA). PCCA is used as smart materials (Holtz and Asher 1997; Holtz et al 1998) for various applications such as in photonics (Lee and Asher 2000; Reese et al 2004), the sensing of metal ions, $\mathrm{pH}$ sensor and sensors for biologically important analytes like glucose and creatinine etc (Reese et al 2001; Alexeev et al 2003; Sharma et al 2004). The degree of self-assembly of the colloidal particles to create CCA has correlation with particle size, surface charge density and ionic strength of the particle medium (Reese et al 2000). Therefore, it is absolutely necessary to fine tune the particle size and the charge density of the colloidal particles as per the needs of the applications. Our aim in this paper is to synthesize copolymer colloidal particles with tunable particle size and charge density which can be utilized to develop PCCA based smart materials.

The properties of the polymer colloidal particles can be fine tuned by varying the sizes of the colloidal particles. In case of emulsion polymerization technique particle size has been shown to vary depending upon the concen- 
tration of emulsifier, initiator, monomer, stirring speed and size of the stirrer blade and temperature. Among all these parameters emulsifier and initiator concentration variation gives rise to particles with a narrow molecular weight and higher monodispersity. Andre and Henry (1998) employed sulfopropylmethacrylate (SPM) as an ionogenic monomer and showed that at constant ionogenic monomer concentration particle size has direct correlation with ionic strength and at constant ionic strength particle size decreases with the concentration of ionogenic monomer. In a recent paper, we have shown that particle size can be varied very sharply by varying the surfactant concentration below the critical micellar concentration of the emulsifier (heterogeneous particle nucleation) and particle size variation can also be achieved by varying the initiator concentration (Arunbabu et al 2008). Here, in this report we are presenting synthesis of highly charged cross linked poly (styrene-co-divinylbenzene-cosulfopropyl methacrylate) colloidal particles of various sizes $(\sim 20-100 \mathrm{~nm})$ using emulsion polymerization method. This report benefitted from the numerous previous works on the synthesis of functionalized monodisperse latex particles (Reese et al 2000; Reese and Asher 2002; Juang and Krieger 1976; Kim et al 1989, 1992; Sunkara et al 1994).

\section{Experimental}

\subsection{Materials}

Divinyl benzene (DVB, Aldrich), 3-sulfopropyl methacrylate (SPM, Poly Sciences), sodium dodecyl sulfate (SDS, Merck), sodium bicarbonate (Sisco) and ammonium per sulfate (APS, Merck) were used as received.

\subsection{Synthesis of poly(styrene-co-divinylbenzene-co- sulfopropyl methacrylate) colloidal particles}

Poly(styrene-co-divinylbenzene- $c o$-sulfopropylmethacrylate), hereafter poly (S-co-DVB-co-SPM), colloidal particles were synthesized by emulsion polymerization technique in four neck mercury sealed round bottom flask fitted with a reflux condenser, teflon stirrer powered by a high-torque mechanical stirrer, nitrogen and reagent inlet. The temperature was maintained by placing the reaction vessel in a controlled temperature oil bath. The reaction vessel was charged with previously deoxygenated $75 \mathrm{ml}$ of double distilled water and $0 \cdot 1 \mathrm{~g}$ of sodium bicarbonate was added. A stirring rate of $360 \mathrm{rpm}$ and a nitrogen blanket were maintained throughout the course of the reaction. The buffer solution was deoxygenated by bubbling with nitrogen for $40 \mathrm{~min}$. After thorough de-oxygenation, the required amount of SDS dissolved in $5 \mathrm{ml}$ of water was added and the temperature was increased to $50^{\circ} \mathrm{C}$. Freshly deoxygenated styrene $(33 \mathrm{~g}, 0.32 \mathrm{~mol})$ and
DVB $(1.65 \mathrm{~g}, 13 \mathrm{mmol})$ were added at a rate of $4 \mathrm{ml} / \mathrm{min}$ using pressure equalizer. The 3-sulfopropylmethacrylate ( $2 \mathrm{~g}, 8 \mathrm{mmol})$ was dissolved in $5 \mathrm{ml}$ of water and injected 10 min after the addition of styrene and DVB. The temperature was increased to $70^{\circ} \mathrm{C}$. After equilibration for $30 \mathrm{~min}$, required amount of ammonium persulfate dissolved in $5 \mathrm{ml}$ of water was injected into the reaction mixture. The reaction was refluxed for 3-4 h. We have varied both the emulsifier/surfactant (SDS) and initiator (APS) concentration keeping all other reaction parameters constant in the polymerization mixture and various reactions recipe are presented in table 1 .

\subsection{Purification of poly(S-co-DVB-co-SPM) colloidal particles}

The polymer formed at the end of the reaction appeared milky white colloidal solution which was filtered through a glass wool. The filtered solution was centrifuged for $40 \mathrm{~min}$ at $45000 \mathrm{rpm}$ at $15^{\circ} \mathrm{C}$ in an ultra centrifuge. The residue (solid white mass) was thoroughly dispersed in double distilled water with the help of sonication and mixing in a vortex motor. Centrifugation and dispersion process were repeated 3-4 times to remove all impurities from the colloidal particles (Ford et al 1994). The colloidal solution was taken in a culture tube and ion-exchange resin (Bio Rad mixed bed; AG501-X8 used as received) was added and placed on a vertical rotor. After 1-2 days of stirring the colloidal solutions were exhibiting bluish violet colour indicating the formation of self assembled array of colloidal particles.

\subsection{Characterization of poly (S-co-DVB-co-SPM) colloidal particles}

The sizes of the colloidal copolymer particles were measured from the microscopic images obtained from the atomic force microscopy (AFM, model: Solver Pro M of NT-MDT) working in semi-contact mode. A micro cantilever with a spring constant of $10 \mathrm{~N} / \mathrm{m}$ was used to scan the samples. The colloidal juice was diluted with water and a drop was placed on a glass substrate and then imaged in AFM. Particle size was measured from the images obtained from the AFM study. The particle charge density and charges per particle were calculated by measuring the volume fraction and $\mathrm{H}^{+}$concentration of the colloidal solution. Volume fraction of colloidal solution was measured gravimetrically in triplicate. The amount of $\mathrm{H}^{+}$ion in the colloidal juice was measured by potentiometric acid-base titration using an AutoTitrator (Titrino 701) instrument (Masui and Watillon 1975; Roberts et al 1998). The colloidal juice was titrated against the $0 \cdot 1(\mathrm{~N}) \mathrm{NaOH}$ solution and $\mathrm{H}^{+}$ion concentration was calculated from the potentiometric titration curve. 
Table 1. Variation of emulsifier and initiator concentration in the reaction.

\begin{tabular}{|c|c|c|c|c|}
\hline Sample no. & SDS (\% wt.) & SDS (mmol) & APS (\% wt.) & APS (mmol) \\
\hline VS129 & $0 \cdot 16$ & 0.69 & $0 \cdot 32$ & 1.75 \\
\hline VS14 & $0 \cdot 32$ & $1 \cdot 39$ & $0 \cdot 32$ & 1.75 \\
\hline VS117 & 0.48 & $2 \cdot 08$ & $0 \cdot 32$ & 1.75 \\
\hline VS128 & 0.64 & $2 \cdot 77$ & $0 \cdot 32$ & 1.75 \\
\hline VS131 & $0 \cdot 32$ & $1 \cdot 39$ & 0.8 & $4 \cdot 39$ \\
\hline VS138 & 0.32 & $1 \cdot 39$ & $2 \cdot 4$ & $13 \cdot 16$ \\
\hline VS141 & $0 \cdot 32$ & $1 \cdot 39$ & $4 \cdot 8$ & $26 \cdot 32$ \\
\hline
\end{tabular}

\subsection{Preparation of PCCA and diffraction studies}

A polymerization mixture consisting of acrylamide $\left(0.1 \mathrm{~g}, 1.39 \mathrm{mmol}\right.$, Sigma Aldrich), $N, N^{\prime}$-methylenebisacrylamide $(0.005 \mathrm{~g}, 0.032 \mathrm{mmol}$, Sigma Aldrich), CCA $[2.00 \mathrm{~g}, 102 \mathrm{~nm}$ particle of poly(S-co-DVB-co-SPM)] latex spheres and $0.1 \mathrm{~g}$ mixed bed resin was prepared. $10 \% \mathrm{w} / \mathrm{v}$ diethoxyacetophenone (DEAP, 8 drops, $100 \mu \mathrm{L}$, $4.96 \mathrm{mmol}$, Sigma Aldrich) in dimethylsulfoxide was added to the mixture. Then the mixture was mixed thoroughly. The polymerization solution from this mixture was withdrawn in syringe carefully using a needle to remove the ion-exchange resin. The reaction mixture was then injected into a cell consisting of two quartz disks, separated by $\sim 125 \mu \mathrm{m}$ thick para films spacer. An UV lamp (Mercury source spot bulb, Sigma Aldrich) operating at $365 \mathrm{~nm}$ was focused on the polymerization cell for $4 \mathrm{~h}$. After polymerization, the cell was open in Millipore water and the PCCA was washed thoroughly with water. A diffracting PCCA was obtained.

All diffraction measurements were carried out in reflectance mode using an Ocean Optics fibre optic spectrometer (USB4000-VIS-NIR).

\section{Results and discussion}

All the copolymers are characterized by using FT-IR spectroscopy. A representative FT-IR spectrum along with the polymer structure and the peaks assignments is shown in figure 1 . The spectrum clearly indicates the presence of stretching frequencies for all the monomers (styrene, divinyl benzene and sulfopropyl methacrylate) and therefore, we can conclude that the synthesized copolymers consist of all the monomers. In table 2 , we have summarized all the characterization results obtained from the emulsion polymerization reactions (table 1) for various concentrations of surfactant and initiator.

\subsection{Particle size}

3.1a Emulsifier concentration $v$ s particle diameter: The particle size decreases from $48 \mathrm{~nm}$ to $24 \mathrm{~nm}$ with increasing concentration of emulsifier (SDS) in solution and is highly dependent upon the concentration of SDS in the reaction mixture (table 2). The representative AFM images of the poly(S-co-DVB-co-SPM) colloids prepared from different surfactant concentrations are presented in figure 2. We have measured particle sizes of the colloids from the AFM images. A plot of particle diameter vs surfactant concentration for a fixed initiator concentration $(19.49 \mathrm{mM})$ is presented in figure 3 . The particle diameter decreases as the surfactant concentration increases. Lower emulsifier concentration produces very less number of micelles which consequently results in larger colloidal polymer particles (Evans and Wennerstrom 1999). A large number of smaller size micelles are found at high emulsifier concentration. Therefore, with increasing emulsifier concentration the particle size decreases (figures 2 and 3). Also, we have observed that as the emulsifier concentration decreases particles size distribution becomes broad (insets of figure 2). This is due to the agglomeration of the colloidal particles because of the decreased stability of the colloids.

3.1b Initiator concentration vs particle diameter: Particle size can also be tuned by varying the initiator concentration in the polymerization mixture. Figure 4 shows the AFM images of different size particles obtained by changing the initiator concentration in the reaction mixture by keeping emulsifier concentration constant. A plot of particle diameter against the initiator concentration for a fixed surfactant concentration is presented in figure 5. It is clear that particle size increases with increasing initiator concentration. Due to the formation of a large number of free radicals at high initiator concentration the possibility of polymerization termination is very high. In an emulsion polymerization system, the probability of monomer swollen micelle maturing into a particle depends upon several factors including the initiator concentration. For a fixed concentration of emulsifier the number of micelles formed is constant but due to the variance in the initiator concentration the portion of these monomer swollen micelles becoming as a particle depends upon the initiator concentration. Higher initiator concentration leads to higher probability of termination so lesser proportions of micelles are converted into particles which results in bigger particles and a less number of particles. A closer observation of AFM images (figure 4) reveal that particles are not monodisperse and they are agglo- 
Table 2. Characteristic data such as particle size and charge density of all synthesized copolymer colloidal particles.

\begin{tabular}{lcccccc}
\hline Sample no. & SDS $(\mathrm{mM})$ & APS $(\mathrm{mM})$ & $\begin{array}{c}\text { Particle } \\
\text { diameter }(\mathrm{nm})\end{array}$ & $\begin{array}{c}\text { Surface charge } \\
\text { density }\left(\mu \mathrm{C} / \mathrm{cm}^{2}\right)\end{array}$ & $\begin{array}{c}\text { Charges spheres }(\mathrm{Z}) \\
(\overline{\mathrm{e}} / \mathrm{sphere})\end{array}$ & $\begin{array}{c}\text { No. of particles }\left(N_{\mathrm{p}}\right) \\
(\mathrm{no} . / \mathrm{mL}) \times 10^{-15}\end{array}$ \\
\hline VS 129 & 7.71 & 19.49 & 48 & 19.20 & 8675 & 1.019 \\
VS 14 & 15.41 & 19.49 & 39 & 11.32 & 3376 & 1.674 \\
VS 117 & 23.12 & 19.49 & 34 & 1.057 & 239 & 4.76 \\
VS 128 & 30.82 & 19.49 & 24 & 0.837 & 95 & 8.94 \\
VS 131 & 15.41 & 48.72 & 68 & 16.69 & 2439 & 1.269 \\
VS 138 & 15.41 & 146.19 & 79 & 6.024 & 20680 & 0.739 \\
VS 141 & 15.41 & 292.40 & 102 & 12290 & 0.558 \\
\hline
\end{tabular}

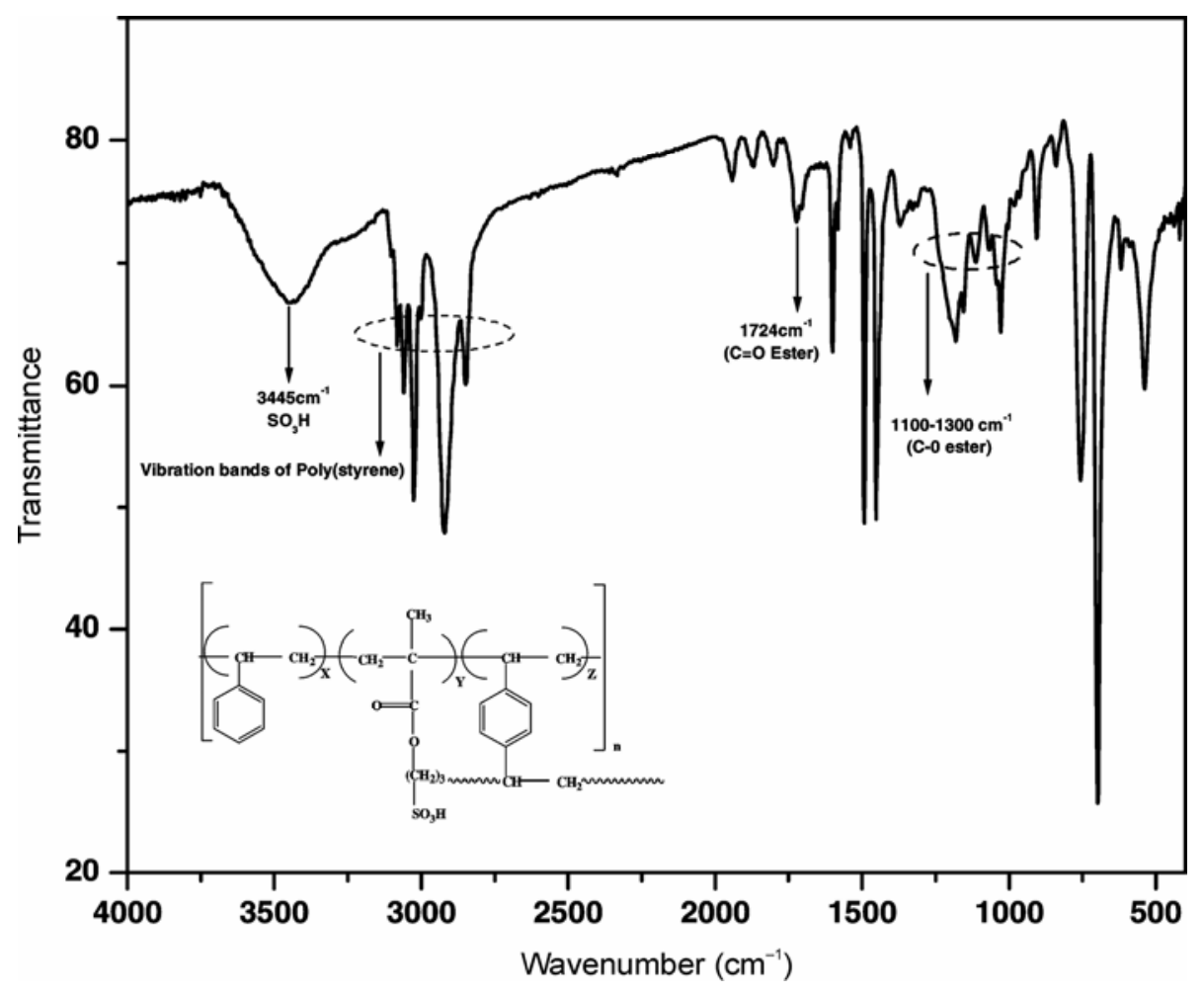

Figure 1. Representative FT-IR spectrum of poly(S-co-DVB-co-SPM) copolymer along with copolymer backbone structure and the peak assignments.

merated. These are due to the decreased electrostatic stability which increases with the increase in particle size. Hence particle size has a direct relationship with initiator concentration which is in agreement with our previous report for similar type of colloids (Arunbabu et al 2008) but not in agreement with the results reported previously by Asher and coworkers (Pan et al 1998) for the fluorinated colloidal particles.

\subsection{Number of particles}

3.2a Number of particles vs surfactant concentration: Harkins' in his classical paper on mechanism of emulsion polymerization (Harkins 1947) proposed that micelles act as a polymerization locus where polymer chain tends to grow in a micelle compartment. Number of particles formed in the yield per unit volume is a function of number of micelles which is a function of concentration of emulsifier molecules. Higher surfactant concentration leads to more number of micelles which consequently mature into smaller polymer particles. From figure 6 it is evident that the number of particles increases with the emulsifier concentration. Hence an inverse relationship between number of particles and particle diameter is expected as presented in the inset of figure 6 . The dependence of the number of particles $\left(N_{\mathrm{p}}\right)$ with the emulsifier/surfactant concentration $(S)$ is obtained from the slope of the straight line in the $\log -\log$ plot of $N_{\mathrm{p}}$ vs $S$ (figure 7) and it is 1.57 . Therefore, the $N_{\mathrm{p}}$ is proportional to $S^{1.57}$. The exponent value is very similar to our earlier 

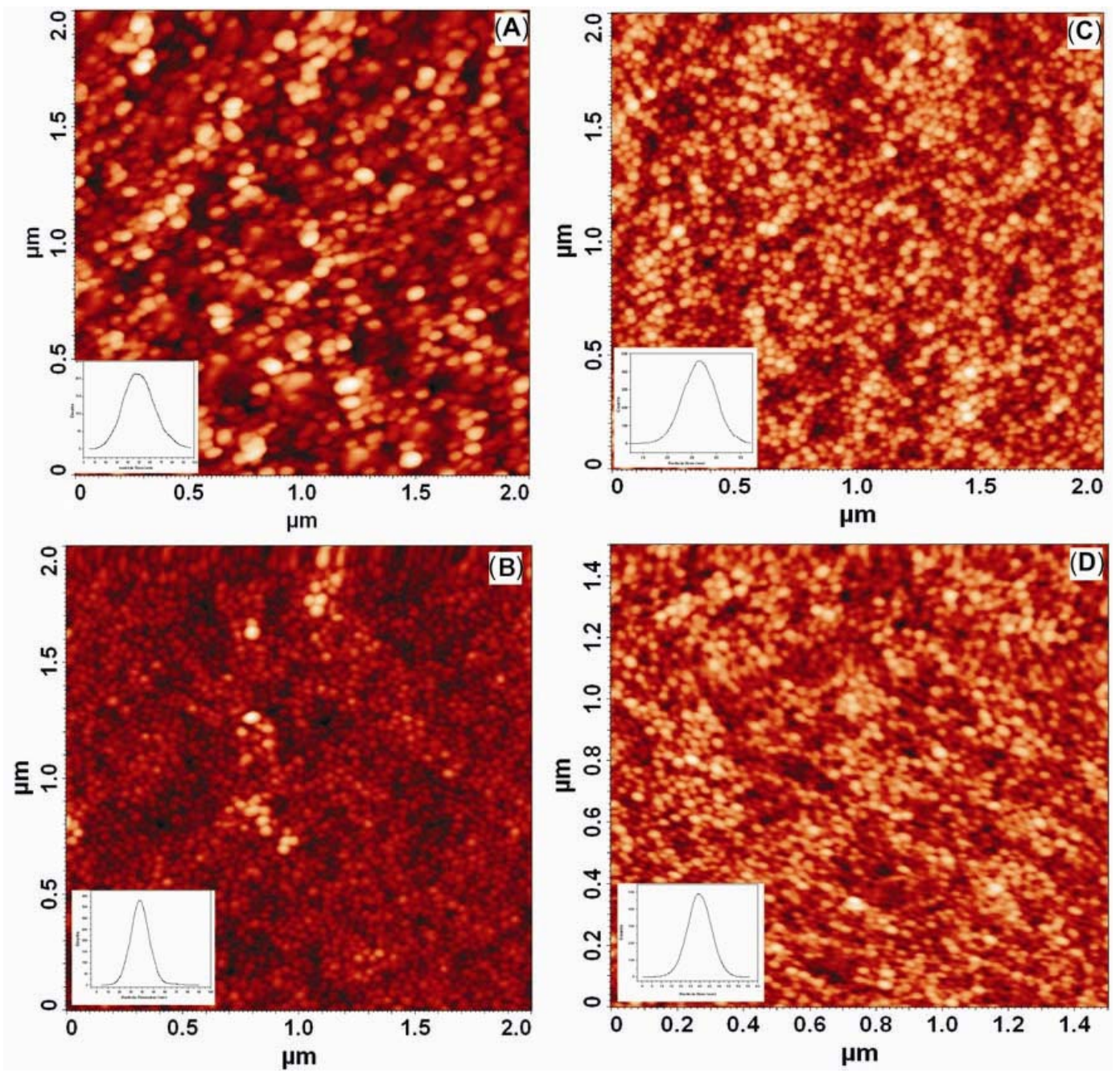

Figure 2. AFM images of colloidal particles prepared by varying SDS concentration: (A) $48 \mathrm{~nm}$, $\mathrm{SDS}=7.71 \mathrm{mM}$ (VS129), (B) $39 \mathrm{~nm}, \mathrm{SDS}=15.41 \mathrm{mM}$ (VS14), (C) $34 \mathrm{~nm}$, SDS = 23.12 mM (VS117) and (D) $24 \mathrm{~nm}, \mathrm{SDS}=30.82 \mathrm{mM}$ (VS128). Particle size distributions are shown in the insets of each micrograph.

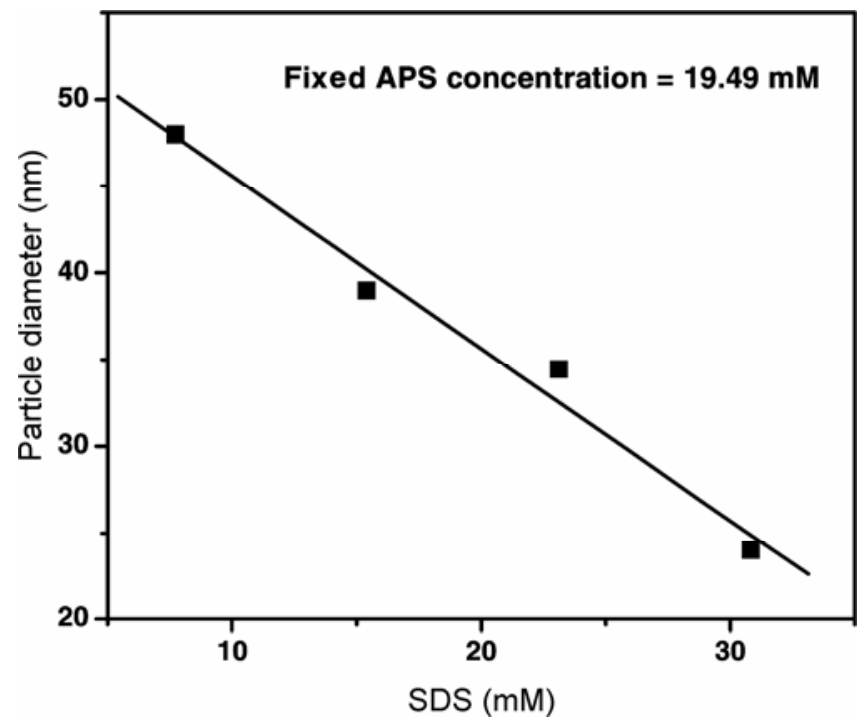

Figure 3. Dependence of particle diameter on SDS (emulsifier) concentration at a fixed APS (initiator) concentration. observation for the similar kinds of colloids (Arunbabu et al 2008). The positive exponent of $N_{\mathrm{p}} \alpha S$ indicates that coagulative's nucleation process takes place during particle formation.

3.2b Number of particles vs initiator concentration: The number of particles decreases with increasing initiator concentration (table 2). The number of micelles is constant for a fixed SDS concentration but polymer particles formed at the end after reaction decides the $N_{\mathrm{p}}$. All the monomer swollen micelles cannot mature as a polymer particle; only a position of them transforms to polymer and this transformation depends upon different parameters such as initiator concentration. The lower initiator concentration increases the probability of transformation of monomer swollen micelles into polymer particles and yields smaller and more number of particles. Thus $N_{\mathrm{p}}$ is inversely proportional to both particle size and initiator concentration (Smith and Ewart 1948). 

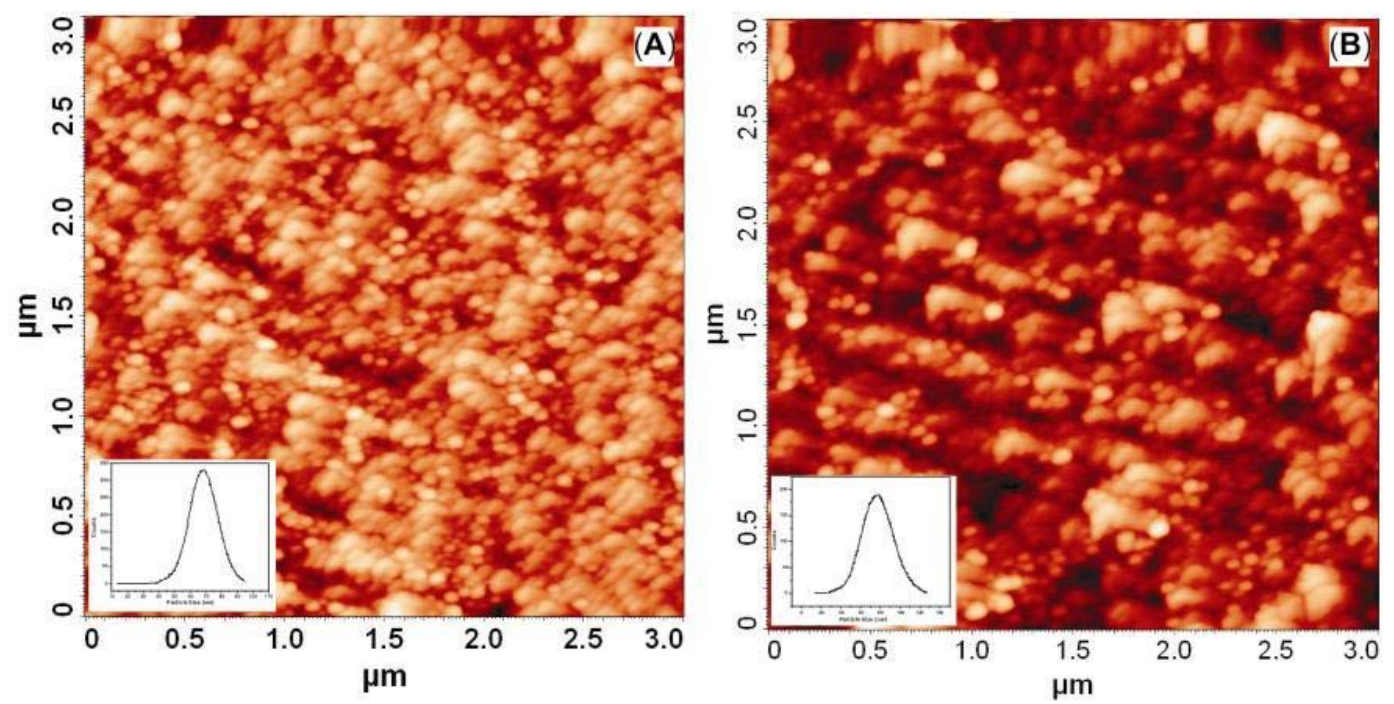

Figure 4. AFM images of poly (S-co-DVB-co-SPM) colloidal particles prepared by varying initiator (APS) concentration for fixed emulsifier concentration $(15.41 \mathrm{mM}) ;(\mathbf{A}) 68 \mathrm{~nm}$, APS $=48.72 \mathrm{mM}$ (VS131) and (B) $79 \mathrm{~nm}$, APS $=146.19 \mathrm{mM}$ (VS138). Particle size distributions are shown in the insets of each micrograph.

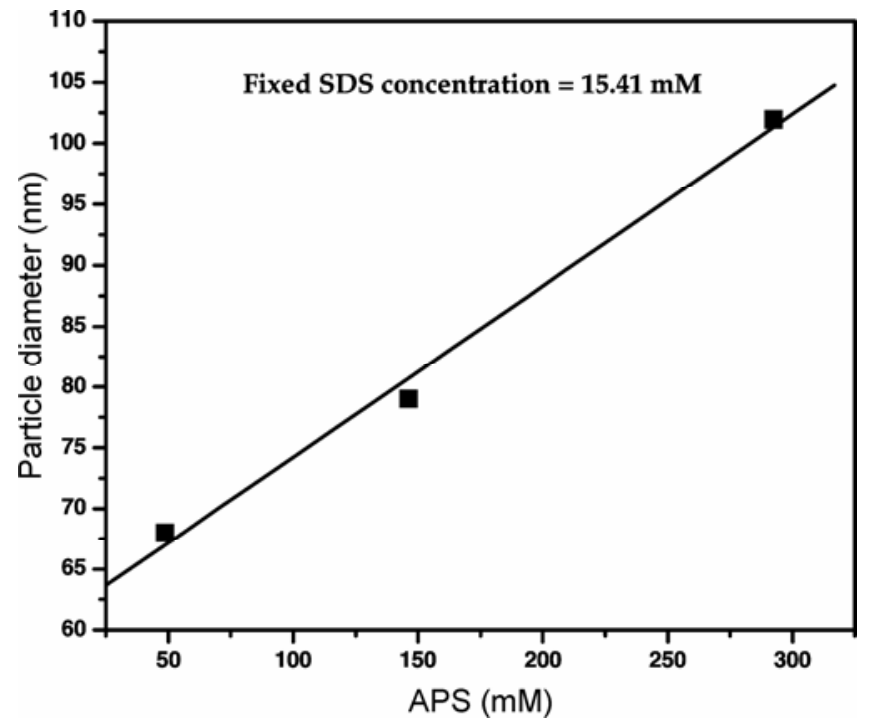

Figure 5. Variation of particle diameter as a function of initiator (APS) concentration at a fixed emulsifier (SDS) concentration.

\subsection{Surface charge density}

Charge on the surface of the particle plays a vital role in designing PCCA based sensor materials. Monodisperse particles with charges on the surface tend to self assemble into non-close packed structures like $b c c$ or $f_{c c}$ in solution. The self assembly is essential in the designing of PCCA based sensing materials. The optimization of the surface charges of the colloidal particles is absolutely necessary because higher charges yield coalescence particles and on the other hand, lower charges result in

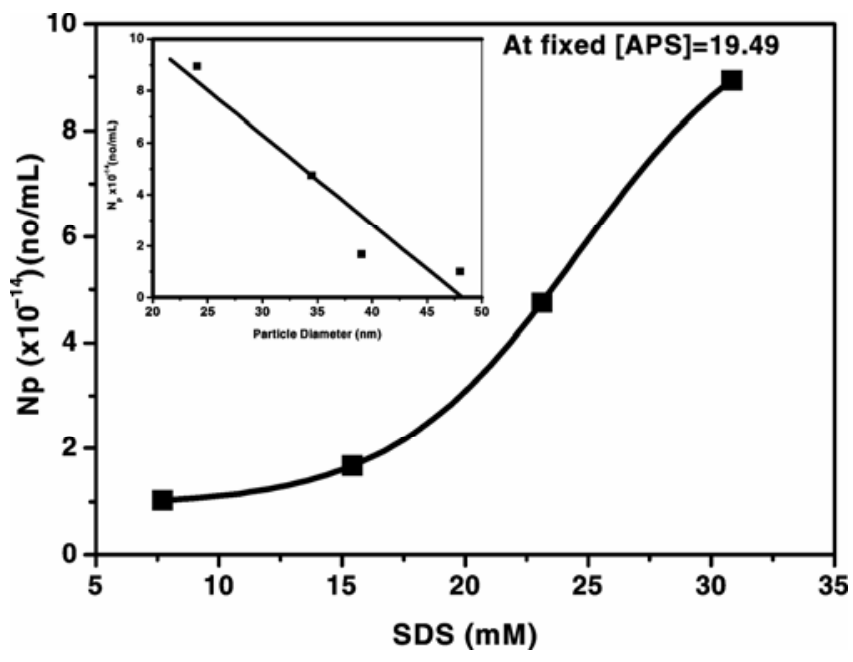

Figure 6. Plot of number of particles vs emulsifier concentration. Inset: $N_{\mathrm{p}}$ vs particle diameter.

agglomerated particles. The role of 3-sulfopropyl methacrylate as an ionic co-monomer is to generate charge on the surface of the spherical particles. The surface charge density i.e. charges per unit area on the surface of a spherical colloidal particle, can be calculated using (1)

$$
\sigma=\frac{F a C_{\mathrm{H}^{+}}(1-\phi)}{3 \phi},
$$

where $\sigma$ is surface charge density, $F$ the Faraday's constant, $C_{\mathrm{H}}^{+}$the concentration of $\mathrm{H}^{+}$ions, $a$ the particle radius and $\phi$ the volume fraction of colloidal juice. Therefore, from (1), one would expect that the charge density of particle would increase with particle diameter. 
Our results are in agreement with (1), as observed in figure 8 . The charge density of the particle increases with particle diameter as is evident in figure 8 . Figure 9 shows that charge density decreases with SDS concentration which is obvious since particle size has an inverse relationship with the SDS concentration. Therefore, it is proved that the surface charge of the colloidal particle is not the contribution from the sulfate groups of SDS; it is due to the sulfate group of ionic co-monomer which is incorporated in the polymer chain. If the surface charge contribution would have come from the SDS then the charge density should have been increased with increasing SDS concentration instead of decreasing with increasing SDS concentration (figure 9). Hence, from the above results it is clearly demonstrated that the origin of the

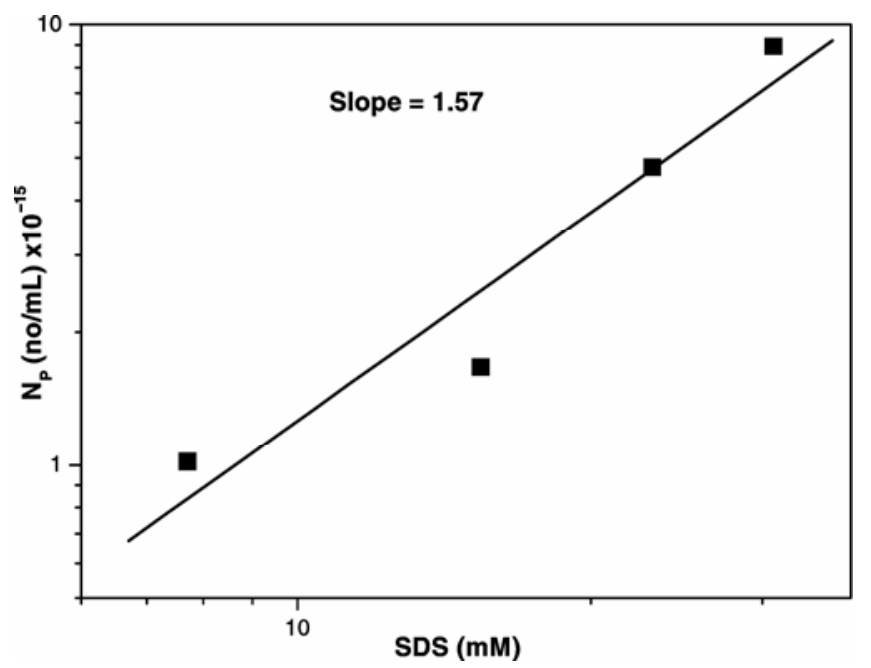

Figure 7. Log-log plot of $N_{\mathrm{P}}$ vs $S$; slope of the solid line is 1.57 .

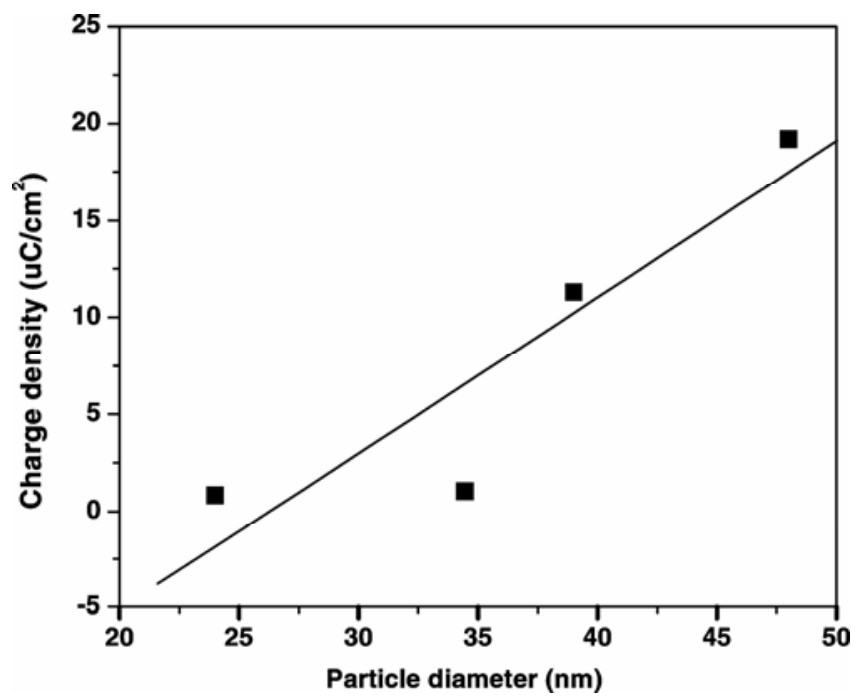

Figure 8. Plot of charge density vs particle diameter (at constant APS concentration). charges on the colloidal particles is the contribution of the ionic co-monomer (sulfopropyl methacrylate) present in the copolymer.

\subsection{Charges per particle}

The number of charges per particle $(Z)$ can be calculated dividing total charge $(Q)$ on the surface of the particle by the electronic charge. The total charge $(Q)$ of the particle is the summation of electron charges on the surface of the sphere. Total charge $(Q)$ on the sphere can be calculated by

$$
Q=\sigma \times 4 \pi a^{2},
$$

where $\sigma$ is the charge density and $a$ the particle radius. Since the particle is spherical the surface area will be

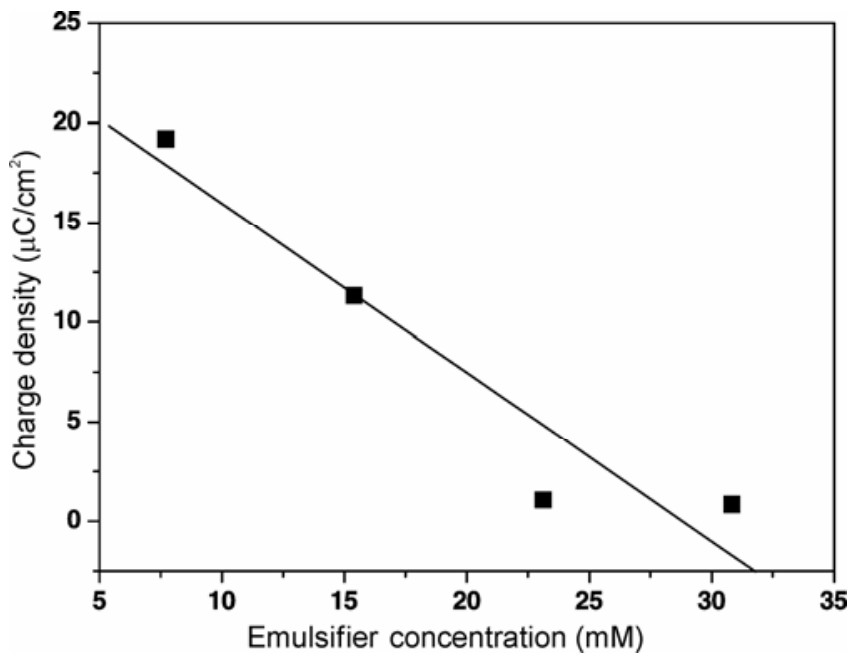

Figure 9. Plot of charge density vs emulsifier concentration (at constant APS concentration).

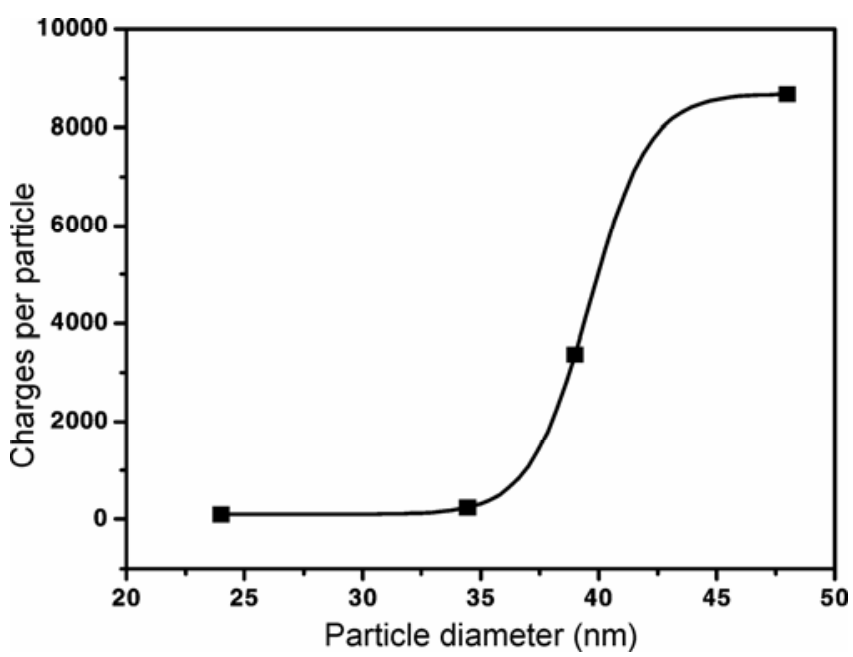

Figure 10. Plot of charges per particle vs particle diameter (at constant initiator concentration). 
$4 \pi a^{2}$. Therefore, the number of charges per particle $(Z)$ is expressed by (3)

$$
z=\frac{\sigma \times 4 \pi a^{2}}{1.602 \times 10^{-19}},
$$

where $1.602 \times 10^{-19}$ Coulomb is the electronic charge. From (3) one would expect a direct correlation between particle diameter and the charges per particle. Charges per particle increases slowly followed by a sudden jump after a certain diameter and attain saturation with the particle diameter as seen in figure 10. Since particle size has an inverse relationship with emulsifier concentration charges per particle rapidly decreases with emulsifier concentration as observed in figure 11. Therefore, one

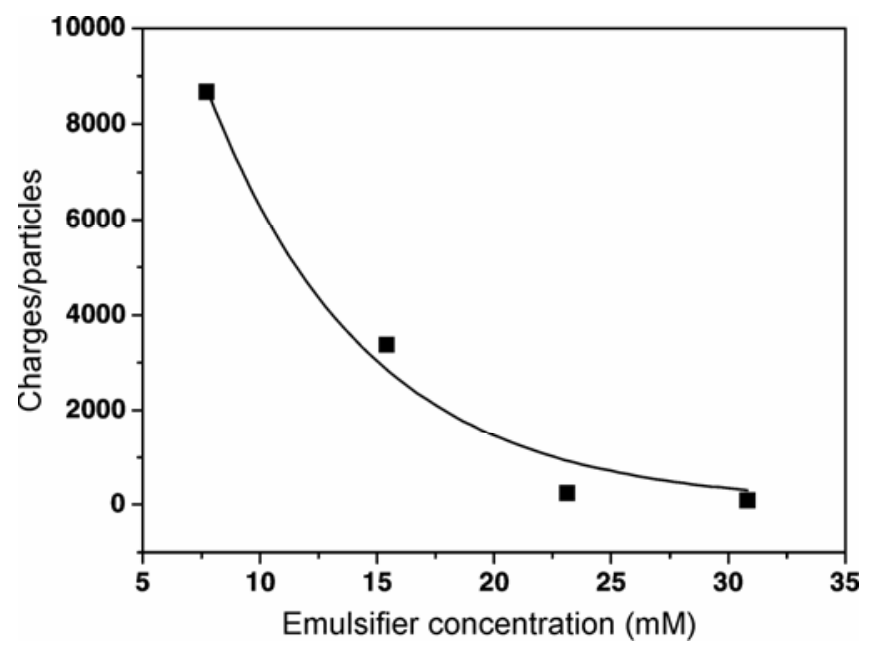

Figure 11. Plot of charges per particle vs emulsifier concentration (at constant initiator concentration).

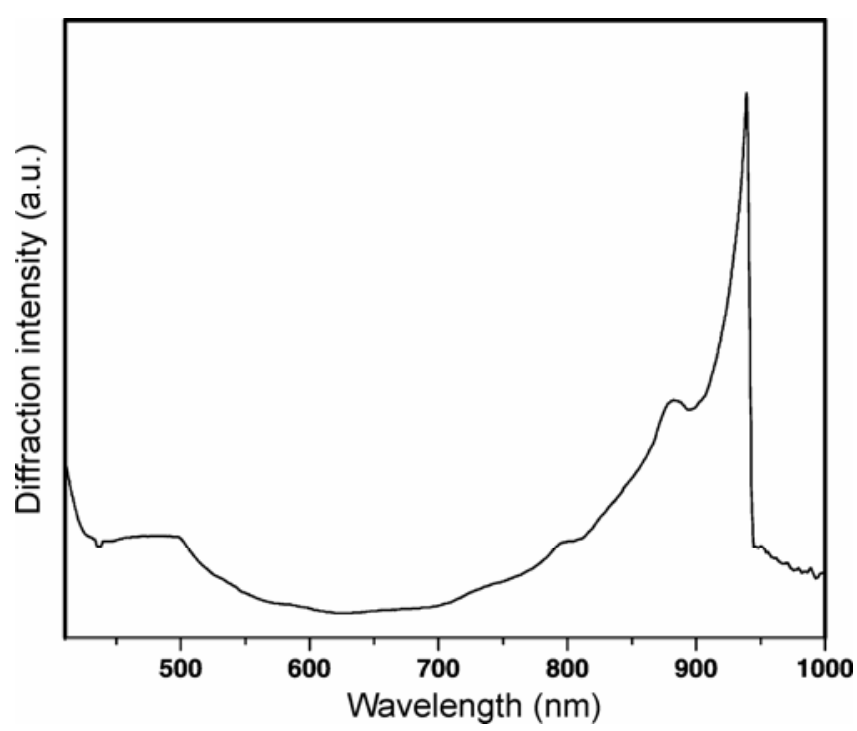

Figure 12. Diffraction spectrum (reflectance spectrum) of the PCCA obtained from the poly(S-co-DVB-co-SPM) colloidal particles. can tune the charges on the particle surface sharply varying the emulsifier concentration.

\subsection{Diffraction studies of PCCA}

After the purification of the poly(S-co-DVB-co-SPM) copolymer colloidal particles we have observed a bright diffracting colour. This indicates that the colloids are self assembled and resulting in the crystalline colloidal array (CCA) (Krieger and O'Neil 1968; Hiltner and Krieger 1969; Hiltner et al 1971; Carlson and Asher 1984; Aastuen et al 1986; Rundquist et al 1989, 1991; Asher et al 1994). We have imbibed this CCA inside the polymer hydrogel matrix by photo polymerizing this copolymer colloidal particles (CCA) with acrylamide and $N, N^{\prime}-$ methylenebisacrylamide. This CCA imbibed hydrogel is known in the literature as polymerized crystalline colloidal array (PCCA) (Holtz and Asher 1997; Holtz et al 1998). The diffraction spectrum (reflectance spectrum) of the PCCA is presented in figure 12. Two diffraction peaks are observed from the PCCA (figure 12). The sharp peak at higher wavelength $(920 \mathrm{~nm})$ is the primary diffraction peak of the CCA and the broad peak $(460 \mathrm{~nm})$ at the half wavelength of the primary diffraction peak is due to second order diffraction of the CCA.

\section{Conclusions}

We have synthesized highly charged colloidal poly (styrene-co-divinylbenzene- $c o$-sulfopropyl methacrylate) copolymer particles using emulsion polymerization. A series of recipes have been developed for the synthesis of 24-102 nm size colloidal particles. Particle size is highly dependent upon the concentration of both the emulsifier and the initiator. The particle diameter decreases with increasing concentration of emulsifier and increases with increasing concentration of initiator in the reaction mixture. Number of particles increases with the increase in emulsifier concentration whereas decreases with initiator concentration. Number of particles has an inverse correlation with the particle size. Charge density can be varied sharply by varying the emulsifier concentration. The number of charges per sphere increases with the particle diameter. Polymer hydrogel imbibed with these copolymer colloidal particles diffracts the light.

\section{Acknowledgements}

We gratefully acknowledge financial support from CSIR (contract grant number (1995)/05/SMR-II). One of the authors (DA) also thanks UGC, New Delhi, for a Senior Research Fellowship.

\section{References}

Aastuen D J W, Clark N A, Cotter L K and Ackerson B J 1986 Phys. Rev. Lett. 571733 
Alexeev V L, Sharma A C, Goponenko A V, Das S, Lednev I K, Wilcox C S, Finegold D N and Asher S A 2003 Anal. Chem. 752316

Andre A and Henry F 1998 Colloid Polym. Sci. 2761061

Arunbabu D, Sannigrahi A and Jana T 2008 J. Appl. Polym. Sci. 1082718

Asher S A, Holtz J, Liu L and Wu Z 1994 J. Am. Chem. Soc. 1164497

Carlson R J and Asher S A 1984 Appl. Spectrosc. 38297

Evans D F and Wennerstrom H 1999 The colloidal domain: where physics, chemistry, biology and technology meet (New York: Wiley-VCH), 2nd edn

Fitch R M 1997 Polymer colloids (San Diego: Academic Press)

Harkins W D 1947 J. Am. Chem. Soc. 691428

Hiltner P A and Krieger I M 1969 J. Phys. Chem. 732386

Hiltner P A, Papir Y S and Krieger I M 1971 J. Phys. Chem. 75 1881

Holtz J H and Asher S A 1997 Nature 389829

Holtz J H, Holtz J S W, Munro C H and Asher S A 1998 Anal. Chem. 70780

Joannopoulos J D, Meade R D and Winn J N 1995 Photonic crystals: molding the flow of light (Princeton: Princeton University Press)

Juang M S D and Krieger I M 1976 J. Polym. Sci.: Polym. Chem. Ed. 142089

Kim J H, Chainey M, El-Aasser M S and Vanderhoff J W 1989 J. Polym. Sci. Part A: Polym. Chem., 273187

Kim J H, Chainey M, El-Aasser M S and Vanderhoff J W 1992 J. Polym. Sci. Part A: Polym. Chem. 30171

Krieger I M and O'Neil F M 1968 J. Am. Chem. Soc. 903114

Lee K and Asher S A 2000 J. Am. Chem. Soc. 1229534
Luck V W, Kleir M and Wesslau H 1963 Ber. Bunsenger. Phys. Chem. 6775

Masui J S and Watillon A 1975 J. Colloid. Interface Sci. 52479

Odian G 2004 Principles of polymerization (New York: WileyInterscience) 4 th edn, Ch. 4

Pan G, Kesavamoorthy R and Asher S A 1997 Phys. Rev. Lett. 7820

Reese C E and Asher S A 2002 J. Colloid Interface Sci. 248 41

Reese C E, Guerrero C D, Weissman J M, Lee K and Asher S A 2000 J. Colloid Interface Sci. 23276

Reese C E, Baltusavich M E, Keim J P and Asher S A 2001 Anal. Chem. 735038

Reese C E, Mikhonin A V, Kamenjicki M, Tikhonov A and Asher S A 2004 J. Am. Chem. Soc. 1261493

Roberts J M, Linse P and Osteryoung J G 1998 Langmuir 14 204

Rundquist P A, Photinos P, Jagannathan S and Asher S A 1989 J. Chem. Phys. 914932

Rundquist P A, Kesavamoorthy R, Jagannathan S and Asher S A 1991 J. Chem. Phys. 951249

Smith W V and Ewart R 1948 J. Chem. Phys. 16592

Soukoulis C M (ed.) 1996 Photonic bandgap materials (Dordrecht: Kluwer)

Soukoulis C M 1999 J. Lightwave Technol. 17

Sharma A C, Jana T, Kesavamoorthy R, Shi L, Virji M A, Finegold D N and Asher S A 2004 J. Am. Chem. Soc. 126 2971

Sunkara H B, Jethmalani J M and Ford W T 1994 J. Polym. Sci. Part A: Polym. Chem. 321431

Xia Y, Gates B, Yin Y and Lu Y 2000 Adv. Mater. 1210 